\title{
miR-378 functions as an onco-miRNA by targeting the ST7L/Wnt/ק-catenin pathway in cervical cancer
}

\author{
SHENGJIE LI $^{1 *}$, FENGXIA YANG $^{2 *}$, MEIYAN WANG ${ }^{3 *}$, WENJUN CAO $^{1}$ and ZHEN YANG ${ }^{4}$ \\ ${ }^{1}$ Department of Clinical Laboratory, Eye and ENT Hospital, Shanghai Medical College, Fudan University, Shanghai 200031; \\ ${ }^{2}$ Department of Clinical Laboratory, Xintai Affiliated Hospital of Taishan Medical University, Xintai, Shandong 271200; \\ Departments of ${ }^{3}$ Clinical Therapeutics and Diagnostics (Theranostics), School of Pharmacy, and ${ }^{4}$ Pathogen Biology, \\ Basic Medical School, Tianjin Medical University, Tianjin 300070, P.R. China
}

Received April 5, 2017; Accepted August 25, 2017

DOI: 10.3892/ijmm.2017.3116

\begin{abstract}
Upregulation or downregulation of microRNAs (miRNAs) has been identified in human cervical cancer (CC). However, the character and function of miR-378 in CC remains unknown. In the present study, the authors demonstrated that miR-378 was upregulated in CC used the reverse transcriptasequantitative polymerase chain reaction (RT-qPCR) assay, and promoted cell proliferation by accelerating the progress of cell cycle and repressing cell apoptosis in CC cells. The predicted target genes of miR-378 were determined by enhanced green fluorescent protein (EGFP) reporter assays, RT-qPCR assay and western blot analysis. miR-378 suppressed the expression of suppression of tumorigenicity 7-like (ST7L) by targeting the 3'-untranslated region (3'-UTR) of ST7L mRNA in HeLa and SiHa cells. ST7L was downregulated in CC using the RT-qPCR assay, and the malignant phenotype of HeLa and SiHa cells were inhibited by ST7L overexpression. In addition, miR-378 activated the Wnt/ $\beta$-catenin pathway by targeting ST7L in CC cells. In short, miR-378 functions as an oncomiRNA by directly downregulating ST7L mRNA and protein level in HeLa and SiHa cells, and serves important roles in the malignancy of CC.
\end{abstract}

\section{Introduction}

Cervical cancer (CC) is one of the most familiar cancers and a leading cause of cancer-associated mortality in women (1). Although the pathological examination of cervical cancer tissue

Correspondence to: Dr Zhen Yang, Department of Pathogen Biology, Basic Medical School, Tianjin Medical University, 22 Qixiangtai Road, Tianjin 300070, P.R. China

E-mail: yangzhen_tmu@163.com

*Contributed equally

Key words: microRNA-378, ST7L, cervical cancer, cell cycle, cell apoptosis, Wnt/ $\beta$-catenin and improved early diagnosis have significantly reduced the morbidity and mortality of this disease in the past decades $(2,3)$, it remains an urgent problem in most developing countries due to poor medical services. Up to now, the common treatments for $\mathrm{CC}$ are still surgical procedures and radiotherapy. In addition, patients who experience metastasis or recurrence are remedied by chemotherapeutics (4). Although the certain molecular mechanisms of cervical carcinogenesis have been investigated (5), the explicit developmental process of cervical carcinogenesis is still mostly indistinct.

MicroRNAs (miRNAs or miRs) include a number of endogenous small non-coding RNAs (approximately 18-22 nucleotides) that serve important roles in controlling gene-targeted expression at the post-transcriptional level by degradation of mRNA or inhibition of translation (6). Previously, miRNAs have been reported as important regulators of many physiological and pathological processes, including cell proliferation, cell differentiation, cell migration and invasion, cell apoptosis and tumor metastasis. miRNAs are reported to regulate target gene expression by binding to their 3'-untranslated regions (3'-UTRs) $(7,8)$. Previous studies have indicated that miR-378 is upregulated and seems to function as an oncogene in ovarian cancer (9), liver cancer (10), human breast cancer (11), nasopharyngeal carcinoma (12), acute myeloid leukemia (13), NSC differentiation (14), human colorectal cancer (15), gastric cancer (16) and renal cell carcinoma (17). However, the underlying clinical roles and characteristic molecular mechanism of miR-378 in CC remain unclear.

Suppression of tumorigenicity 7-like (ST7L) was identified based on its similarity to the ST7 tumor suppressor gene (18). ST7L is downregulated in different cancers, including gastric cancer (19), glioma (20), ovarian cancer (21) and hepatocellular carcinoma (22). For example, Chen et al (20) demonstrated that the deletion of miR-24 suppressed $\beta$-catenin/Tcf- 4 transcription activity by targeting ST7L in glioma. In addition, Yang et al (21) reported that upregulated miR-23a promoted cell malignant phenotype by targeting ST7L in epithelial ovarian cancer cells. Recently, Zhuang et al (22) indicated that ST7L could interact with the carboxyl terminal region of AKT and suppress AKT/GSK3/ $/ \beta$-catenin pathway in HCC cells. However, the significance of ST7L in CC and the underlying mechanism has not been elucidated. 
In the present study, the authors demonstrated that miR-378 might function as an oncogene by promoting cell growth, accelerating the cell cycle and inhibiting cell apoptosis by directly downregulating ST7L in HeLa and SiHa cells. Overexpression of the miR-378-activated Wnt/ $\beta$-catenin pathway in $\mathrm{CC}$. Collectively, these findings may provide insight into tumorigenesis and a potential biomarker for $\mathrm{CC}$.

\section{Materials and methods}

Materials. A total of 27 pairs of human cervical tissue, consisting of human $\mathrm{CC}$ and matched normal cervical tissue from the same patient, were used in the study. Written informed consent was obtained from all enrolled patients, and all relevant investigations were performed according to the principles of the Declaration of Helsinki. The samples were received from the Department of Oncology, Xintai Affiliated Hospital of Taishan Medical University, Taian, China. Total RNA was extracted from the human samples and purified using the miRVana miRNA Isolation kit (Ambion, Thermo Fisher Scientific, Inc., Waltham, MA, USA) according to the manufacturer's instructions. The study was approved by the Ethical Review Committee of Xintai Affiliated Hospital of Taishan Medical University (Ethics approval number: 20150023).

Cell lines and transfection assay. Normal human endocervical epithelial cell lines (Endl/E6E7) was obtained from Shanghai Medical College, Fudan University (Shanghai, China), which were cultured in KER-SFM medium supplemented with $10 \%$ calf serum (Biological Industries, Carlsbad, CA, USA) at $37^{\circ} \mathrm{C}$ with $5 \% \mathrm{CO}_{2}$. Other cervical cancer cells used in the present study were obtained from the American Type Culture Collection (ATCC; Manassas, VA, USA) and were cultivated in RPMI-1640 (Invitrogen, Thermo Fisher Scientific) supplemented with $8 \%$ fetal calf serum (FCS; Biological Industries), $100 \mathrm{U} / \mathrm{ml}$ penicillin, and $100 \mu \mathrm{g} / \mathrm{ml}$ streptomycinat $37^{\circ} \mathrm{C}$ in a $5 \% \mathrm{CO}_{2}$ constant temperature incubator. A transfection assay preceded using Lipofectamine ${ }^{\mathrm{TM}} 2000$ reagent (Invitrogen, Thermo Fisher Scientific) according to the protocol supplied by the manufacturer (Invitrogen).

Plasmid construction. For miR-378, an overexpression vector (pri-miR-378) containing a miR-378 precursor region was amplified from the genomic DNA and inserted into the vector of pcDNA3. Pri-miR-378-S, 5'-CGACGCGTCGGGCTGCG AGGAGTGAGCG-3' and Pri-miR-718-AS, 5'-CCATCGAT GGGAGTTCAAATGGCTTGCTCC-3'. The 2'-O-methylmodified miR-378 antisense oligonucleotide (ASO-miR-378) was commercially synthesized as an inhibitor of miR-378. ASO-miR-378, 5'-CCUUCUGACUCCAAGUCCAGU-3' and ASO-NC, 5'-CAGUACUGUAGUGUAGUACTT-3'. The segment of 3'-UTR of ST7L containing the miR-378 targets was acquired by polymerase chain reaction with gene-specific primers, and then cloned into pcDNA3/enhanced green fluorescent protein (EGFP) following the stop codon of luciferase with BamHI and HindIII sites. ST7L-3'-UTR-S, 5'-CGGGAT TCGGTCAAAGAGAAAGAACTCTAATGTCCAGCTGCTC CATCGA-3' and ST7L-3'-UTR-AS, 5'-CGGAATTCTCGAT GGAGCAGCTGGACATTAGAGTTCTTTCTCTTTGACC-3'. ST7L-3'-UTR-mut-S, 5'-CGGGATTCGGTCAAAGAGAA
AGAAGTATACTGCTGACCTGCTCCATCGA-3' and ST7L3'-UTR-mut-AS, 5'-CGGAATTCTCGATGGAGCA GCTG GTCAGCAGTATACTTTCTCTTTGACC-3'. The full-length sequences of human ST7L cDNA (NM_017744.4) were obtained by reverse transcriptase-quantitative polymerase chain reaction (RT-qPCR) and were cloned into pcDNA3 at $K p n I$ and EcoRI sites. The resulting plasmid was termed pST7L. ST7L-S, 5'-CAGGGGTACCGCCACCATGGCGG ACCGTGGCGGCGTG-3' and ST7L-AS, 5'-CCGGAATTC GCCAGAACTCAAACCTAGGTCTTC-3'.

RNA isolation and detection. Total RNA was extracted with TRIzol reagent (Sigma-Aldrich; Merck KGaA, Darmstadt, Germany) according to the manufacturer's instructions. The quality and integrity of acquired total RNA was evaluated by NanoDrop 2000c; Thermo Fisher Scientific, Inc. (Wilmington, DE, USA) and $1 \%$ gel electrophoresis, respectively. For RT-qPCR, $2 \mu \mathrm{g}$ total RNA was reverse transcribed with miR-378, U6 RT primers, or oligo-dT with M-MLV reverse transcriptase. RT-qPCR was performed with kits, and produced the following reaction: $2 \mu 1 \mathrm{RT}$ products, 5 pmol forward primer, 5 pmol reverse primer, $7.5 \mu 12 \mathrm{X}$ SYBR-Green buffer and nuclease-free water to $15 \mu \mathrm{l}$. The primers used were followed by: miR-378-RT, 5'-GTCGTATCCAGTGCAGGGTCCGAG GTGCACTGGATACGACGCCTTCT-3'; miR-378-forward, 5'-TGCGGACUGGACUUGGAGUC-3'; U6-RT, 5'-GTC GTATCCAGTGCAGGGTCCGAGGTATTCGCACTGG ATACGACAAAATATGGAAC-3'; U6-qPCR-forward, 5'-TGCGGGTGCTCGCTTCGGCAGC-3'; Oligo-dT, 5'-TTTTTTTTTTTTTTTTTT-3'; $\beta$-actin-qPCR-forward, 5'-CGTGACATTAAGGAGAAGCTG-3' and reverse, 5'-CT AGAAGCATTTGCGGTGGAC-3'; Universal reverse qPCR primer, 5'-CCAGTGCAGGGTCCGAGGT-3'; ST7L-qPCR-forward, 5'-CGCGGATCCCCTCTGTGTGT GTGTGTGTAAC-3' and reverse, 5'-CCGGAATTCGCATT CCTGGGCAGGTCGGT-3'.

MTT assay. Cervical cells were seeded into the plates of a 96-well plate at 5,000 cells/well one day prior to transfection. The HeLa and SiHa cells were transfected with pri-miR-378, ASO-miR-378, or the respective control vectors. Cell viability at 24, 48 and $72 \mathrm{~h}$ post-transfection was determined by MTT assay. The absorbance values at $490 \mathrm{~nm}$ were measured via the Quant Microplate spectrophotometer (BioTek Instruments, Inc., Winooski, VT, USA).

Colony formation assay. For the colony formation ability assay, the HeLa and SiHa cells were counted at $24 \mathrm{~h}$ posttransfection and seeded into 24-well plates at 500 cells/well. Culture medium was replaced every $72 \mathrm{~h}$. After approximately two weeks, cells were cleaned with $1 \mathrm{X}$ phosphate-buffered saline (PBS), stained with common crystal violet dye, and colonies containing $>50$ cells were counted.

Prediction of miRNA targets. The hypothetical targets of miR-378 were predicted using TargetScan 7.1, RNAhybrid and microRNA.org.

EGFP reporter assay. The EGFP reporter plasmids with ST7L 3'-UTR or ST7L 3'-UTR-mut were transfected into HeLa and 

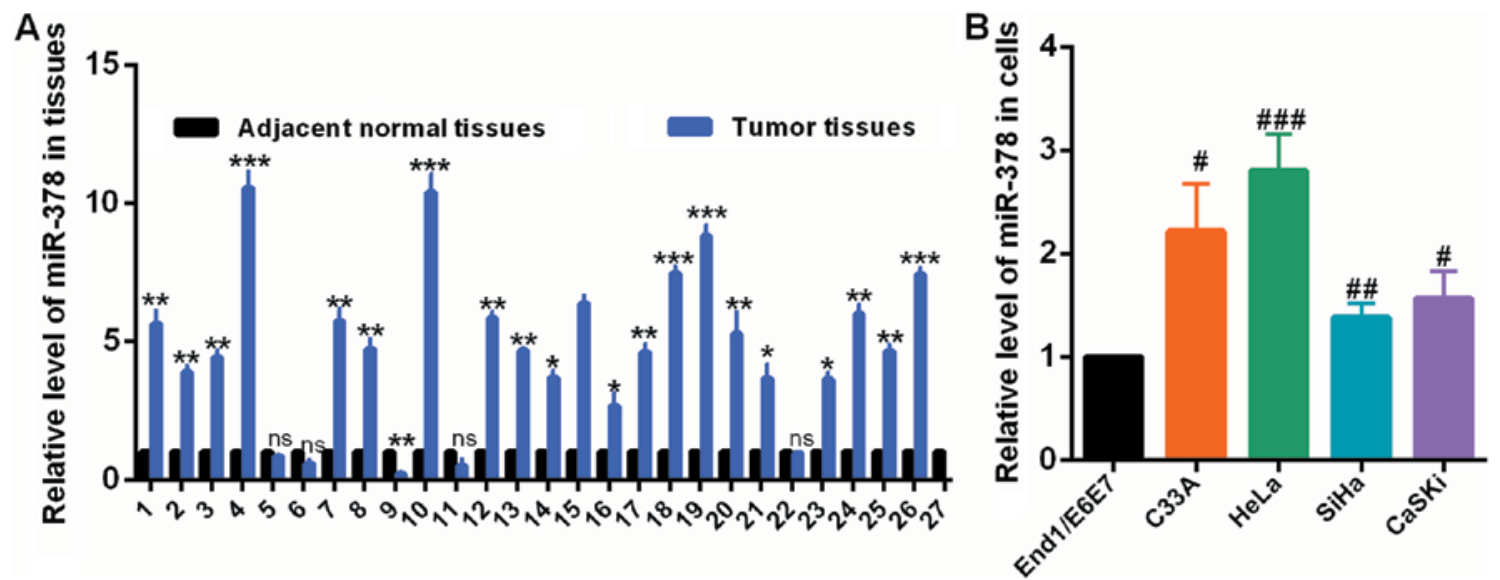

Figure 1. miR-378 was upregulated in CC tissues and CC cells. (A) The levels of miR-378 in CC and normal tissues were examined by RT-qPCR assay. (B) The levels of miR-378 in C33A, HeLa, SiHa, CaSKi cells and End1/E6E7 cells were examined by RT-qPCR assay. Data are presented as mean \pm SD $(n=3)$. "P<0.05, ${ }^{* *} \mathrm{P}<0.01,{ }^{* * *} \mathrm{P}<0.001$ vs. the adjacent normal tissues; ${ }^{\#} \mathrm{P}<0.05,{ }^{\# \#} \mathrm{P}<0.01,{ }^{\# \# \#} \mathrm{P}<0.001$ vs. End1/E6E7 cell line. CC, cervical cancer; miR, microRNA; RT-qPCR, reverse transcription-quantitative polymerase chain reaction.

SiHa cells with Lipofectamine 2000 reagent (Invitrogen; Thermo Fisher Scientific) and RFP expressing plasmid was integrated as a transfection efficiency control. Cells were lysed $48 \mathrm{~h}$ post-transfection, and the intensities of EGFP and RFP fluorescence were determined with a spectrophotometer.

Western blot assay. Cell extracts were cleaned with 1X PBS buffer, prepared with RIPA buffer supplemented with cocktail, and protein concentrations were quantified using the BCA protein assay kit (Beyotime Institute of Biotechnology, Shanghai, China) according to the manufacturer's protocols. Equal amounts of proteins were separated by $10 \%$ SDS-PAGE and subsequently transferred to polyvinylidene difluoride (PVDF) membranes (Millipore, Billerica, MA, USA). The membranes were then blocked with $5 \%$ non-fat milk in Trisbuffered saline with Tween-20 (TBST) for $\sim 2 \mathrm{~h}$, then followed by incubation with the primary antibodies against GAPDH (1:2,000; WL01547; Wanlei Biotech Co., Ltd., Beijing, China) and ST7L (1:1,000; 17567-1-AP; Proteintech Co., Ltd., Wuhan, China) overnight at $4^{\circ} \mathrm{C}$. After washing with TBST, the blots were incubated with horseradish peroxidase (HRP) conjugated secondary antibody (1:5,000; A0216; Beyotime Institute of Biotechnology) at $37^{\circ} \mathrm{C}$ for $1 \mathrm{~h}$. Thereafter, the proteins of interest were visualized using enhanced chemiluminescence (ECL; Wanlei Biotech) and densitometric analysis was performed using Gel-Pro Analyzer system (Beijing Liuyi Instrument Factory, Beijing, China). LabWorks ${ }^{\mathrm{TM}}$ Image Acquisition and Analysis software UVP EC3 (UVP, LLC, Upland, CA, USA) were used to quantify band intensities.

Cell cycle and apoptosis flow cytometric analyses. At $48 \mathrm{~h}$ after transfection, transfected CC cells were harvested by trypsinization and resuspended in cold PBS for analysis. For the analysis of cell cycle, cells stained with propidium iodide (PI) according to the manufacturer's manual. The rate of cell apoptosis was detected using an Annexin V-FITC/PI apoptosis detection kit (Nanjing Kaiji Biotechnology Development Co., Ltd., Nanjing, China). These analyses were conducted according to the protocol provided by Nanjing Kaiji Biotechnology Development.
TOP/FOP flash reporter assays. To assay the transcriptional activity of Wnt pathway, pri-miR-378, pST7L, or pri-miR-378 and pST7L treated cells were co-transfected with either the Wnt signaling reporter TopFlash or the negative control FopFlash according to the protocol (EMD Millipore). HeLa cells were transiently transfected with either $2 \mu \mathrm{g}$ pTopFlash (TCF reporter plasmid) or pFopFlash (mutant, inactive TCF binding site) plasmids (EMD Millipore) and $0.5 \mu \mathrm{g} \mathrm{pSV40-}$ Renilla plasmid as an internal control (Promega Corp., Madison, WI, USA) for $48 \mathrm{~h}$. The Dual-Luciferase reporter assay system (DLR ${ }^{\mathrm{TM}}$ E1910; Promega) was used to assay the firefly and Renilla luciferase activity ratio.

Statistical analysis. Data are presented as the means \pm standard deviation (SD) from at least three independent experiments. Statistical analyses were performed using Student's t-tests. $\mathrm{P}<0.05$ was considered to indicate a statistically significant difference.

\section{Results}

miR-378 is upregulated in human cervical cancer and cervical cell lines. To investigate the role and clinical significance of miR-378 in cervical cancer, the authors first detected the level of miR-378 by RT-qPCR assay in 27 pairs of human cervical tumors and matched normal cervical tissues. Compared with the corresponding non-tumorous counterparts, the miR-378 expression level was significantly upregulated in cervical tumor tissues (Fig. 1A). In addition, RT-qPCR was also used to examine the expression level of miR-378 in HeLa, C33A, SiHa and CaSKi cells, and in End1/E6E7 cells, which is a human normal cervical epithelium cell line. The results demonstrated that miR-378 was obviously elevated in cervical cancer cells compared to End1/E6E7 cells (Fig. 1B). These results identified that miR-378 was upregulated in human cervical cancer.

$m i R-378$ promotes cervical cancer cell growth in vitro. The roles of miR-378 in cervical cancer cells were evaluated by transfection of miR-378 overexpression or knockdown plasmids in HeLa and SiHa cells (Fig. 2A). An MTT assay was 

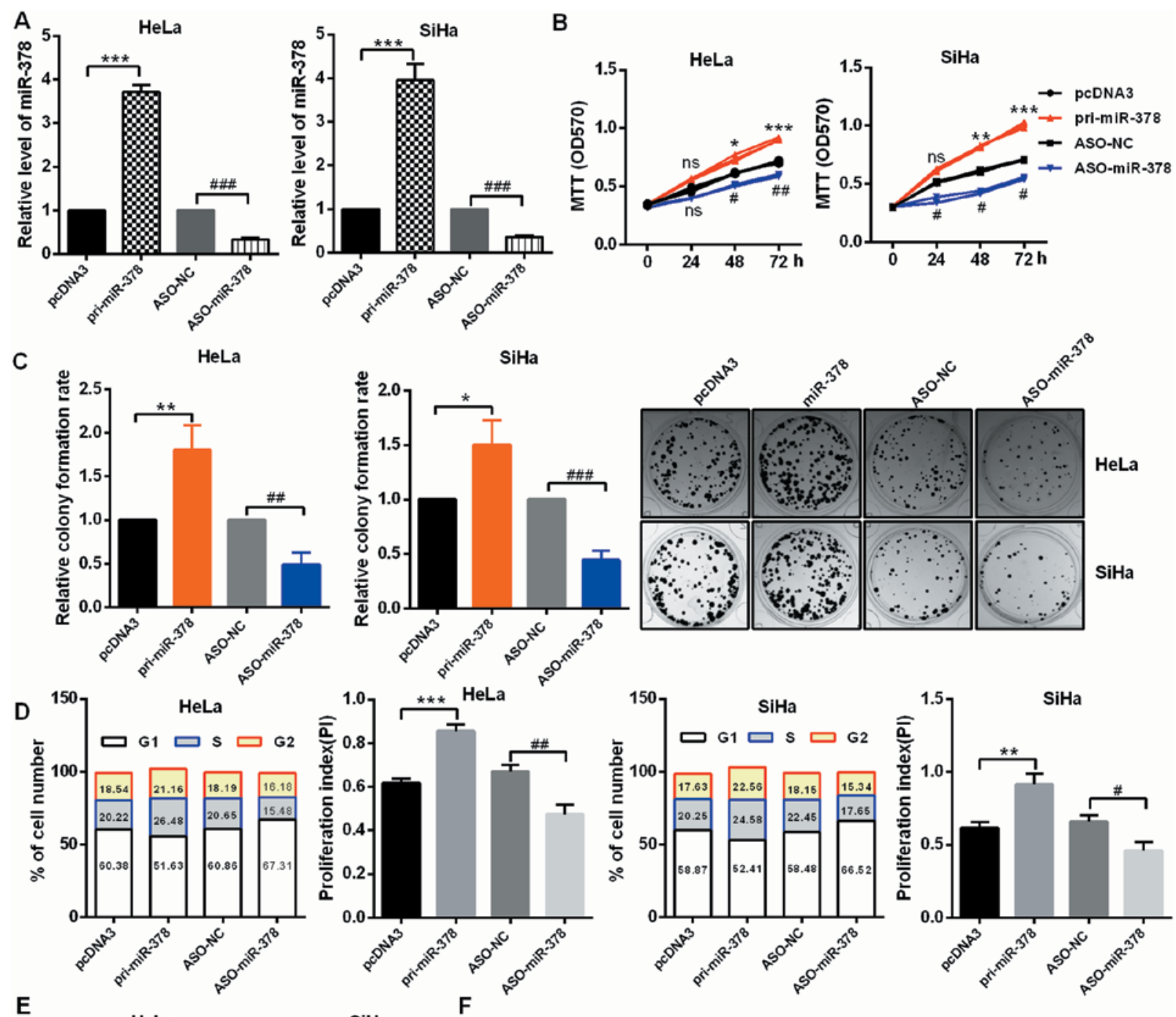

E
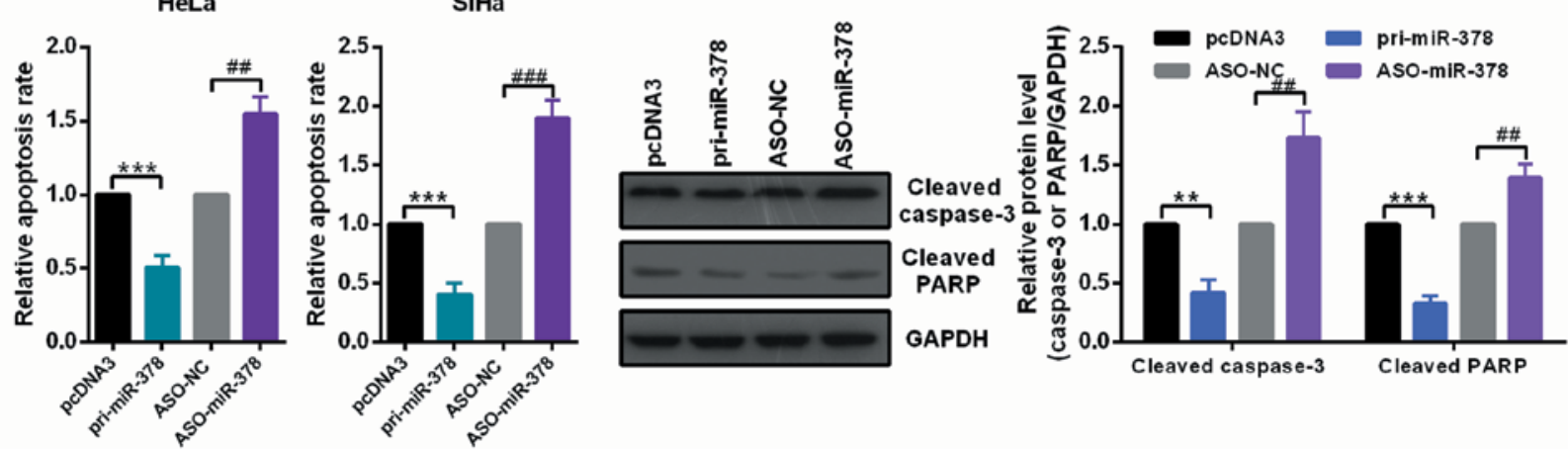

Figure 2. miR-378 served as an onco-miRNA in cervical cancer cells. (A) The efficiency of miR-378 overexpression and knockdown plasmids was confirmed by reverse transcription-quantitative polymerase chain reaction assay. HeLa and SiHa cells were transfected with the pri-miR-378 or ASO-miR-378 and the control groups, respectively. (B) The cell viability of miR-378 on HeLa and SiHa cells were determined by MTT assay. Overexpression of miR-378 promoted cell viability and knockdown of miR-378 inhibited cell viability. (C) Relative colony formation rate of HeLa and SiHa cells with indicated treatment was determined by colony formation assay. Overexpression of miR-378 promoted colony formation ability and knockdown of miR-378 inhibited colony formation ability. Original magnification, x1. (D) Flow cytometry cell cycle assays demonstrated that miR-378 increased the number of HeLa and SiHa cells in the S and G2 phases and decreased the number of HeLa and SiHa cells in G1 phase. Overexpression of miR-378 enhanced the proliferation index. (E) Flow cytometry cell apoptosis assays revealed that miR-378 overexpression suppressed the apoptosis of HeLa and SiHa cells and miR-378 knockdown promoted cell apoptosis. (F) The expression levels of cleaved PARP and caspase-3 were examined by western blot assay in HeLa cells under the described condition. Data are presented as mean $\pm \mathrm{SD}(\mathrm{n}=3) .{ }^{*} \mathrm{P}<0.05,{ }^{* *} \mathrm{P}<0.01,{ }^{* * *} \mathrm{P}<0.001$ vs. the pcDNA3 group; ${ }^{\#} \mathrm{P}<0.05,{ }^{\# \#} \mathrm{P}<0.01,{ }^{\# \#} \mathrm{P}<0.001$ vs. the ASO-NC group. miR, microRNA; ASO, antisense oligonucleotide; $\mathrm{NC}$, negative control; ns, not significant; pri, primary.

then performed to determine cell viability. Data indicated that transfection with pri-miR-378 significantly facilitated the proliferation of HeLa and SiHa cells; on the contrary, ASO-miR-378 transfection in HeLa and SiHa cells remarkably decreased cell viability compared to the controls (Fig. 2B). Furthermore, upregulation of miR-378 increased the relative colony formation rate in HeLa and $\mathrm{SiHa}$ cells, whereas miR-378 downregulation inhibited the colony formation ability of HeLa 


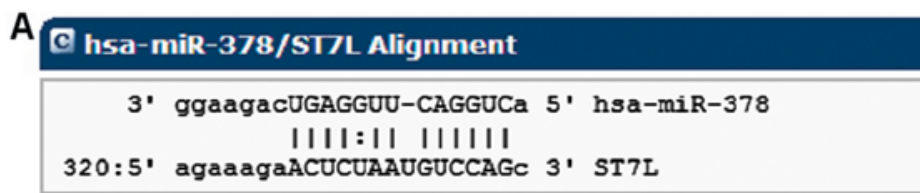

3'GGAAGACUGAGGUU CAGGUCA5' hsa-miR-378

| | || || ||| | |

5'AGAAAGAAGUAUACUGCUGACC3' ST7L-3' UTR-mut

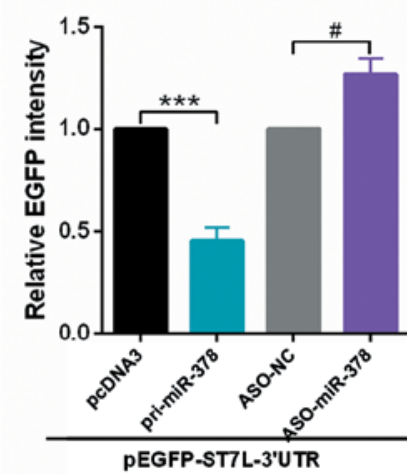

D

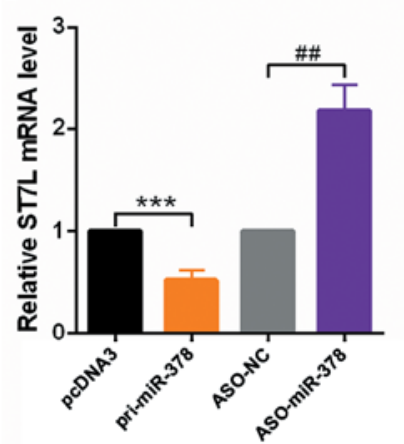

$\mathrm{SiHa}$

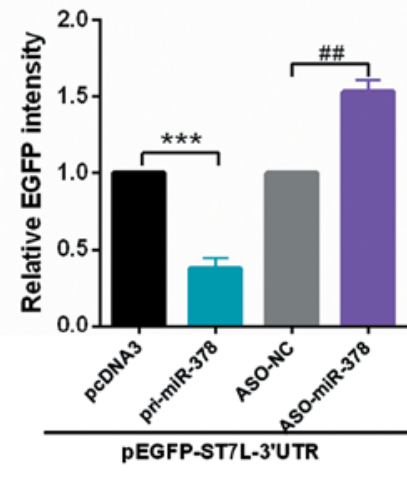

$\mathrm{SiHa}$

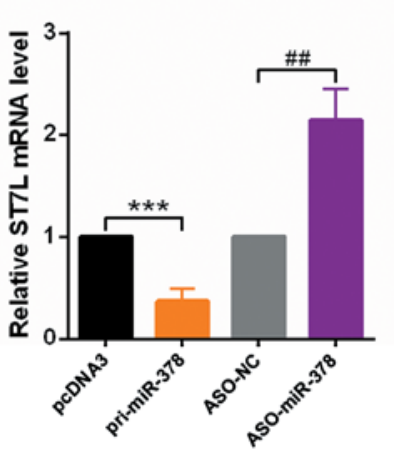

C

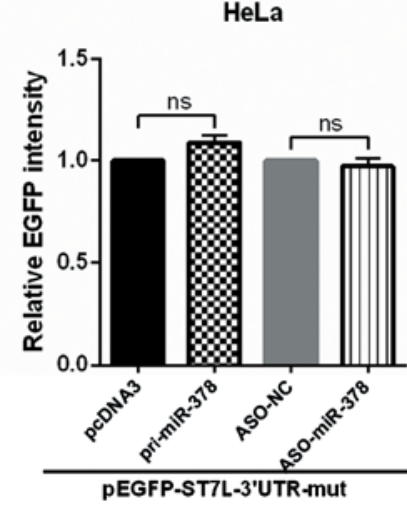

E

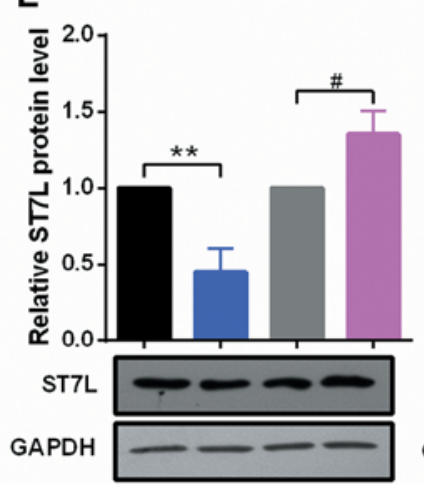

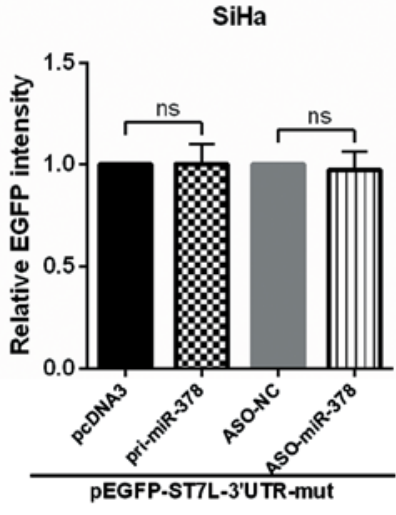

$\mathrm{SiHa}$

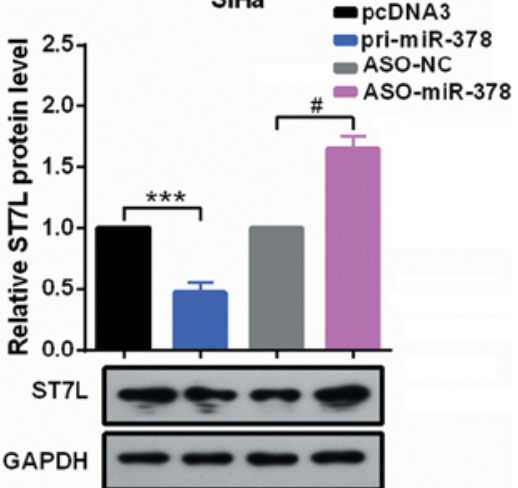

Figure 3. miR-378 directly targets ST7L in cervical cancer cells. (A) The predicted miR-378 binding sites using TargetScan 7.1 in ST7L mRNA 3'-UTR and the sites of ST7L mRNA 3'-UTR-mut is shown. (B and C) HeLa and SiHa cells were co-transfected with pcDNA3/EGFP-ST7L 3'-UTR or 3'-UTR-mut and pri-miR-378 or ASO-miR-378. EGFP intensity was determined by spectrophotometer, and the value of the control group (pcDNA3 or ASO-NC) was set to one. (D) HeLa and SiHa cells were transfected with pri-miR-378 or ASO-miR-378. ST7L mRNA level in HeLa and SiHa cells were measured by reverse transcription-quantitative polymerase chain reaction assay. (E) ST7L protein level in HeLa and SiHa cells transfected with pri-miR-378 or ASO-miR-378 and the respective controls was determined by western blot assay. Data are presented as mean $\pm \mathrm{SD}(\mathrm{n}=3) .{ }^{* *} \mathrm{P}<0.01,{ }^{* * *} \mathrm{P}<0.001$ vs. the pcDNA3 group; ${ }^{\#} \mathrm{P}<0.05$, ${ }^{\# \#} \mathrm{P}<0.01$ vs. the ASO-NC group. ns, not significant; miR, microRNA; 3'-UTR, 3'-untranslated region; mut, mutant; pri, primary ASO, antisense oligonucleotide; EGFP, enhanced green fluorescent protein; NC, negative control.

and SiHa cells (Fig. 2C). These data suggested that miR-378 served as an onco-miRNA in cervical cancer cells.

miR-378 markedly accelerates cell cycle progression and reduces cell apoptosis in CC cells. miR-378 significantly promoted the growth of HeLa and SiHa cells, so it was speculated that miR-378 could accelerate the cell cycle process in cervical cancer cells. The authors reported that overexpression of miR-378 obviously decreased the percentage of cells in the G1 phase and increased the percentage of cells in the S and G2 phase in both HeLa and SiHa cells compared with cells transfected with the controls; however, knockdown of miR-378 increased the percentage of cells in the G1 phase and decreased the percentage of cells in the $\mathrm{S}$ and G2 phase in HeLa and SiHa cells by flow cytometry assay. In addition, cells treated with pri-miR-378 markedly increased the proliferation index of HeLa and SiHa cells, cells treated with ASO-miR-378 decreased the proliferation index of HeLa and SiHa cells, respectively (Fig. 2D). Thus, the authors demonstrated that miR-378 might promote the proliferation of cervical cancer cells by accelerating cell cycle transition. To investigate whether cell apoptosis took part in the miR-378-induced proliferative effect, the relative cell apoptosis rates of HeLa and SiHa cells were detected by flow cytometry cell apoptosis assay. Fig. 2E indicated that the relative apoptotic rate of $\mathrm{HeLa}$ and $\mathrm{SiHa}$ cells was significantly lower in the miR-378-treated group than that in the control groups. In addition, the noted effect of miR-378 on apoptosis was confirmed, as decreased activating cleavage of PARP and caspase-3 was induced in the cervical cancer cells that overexpressed miR-378 (Fig. 2F).

miR-378 directly targets ST7L in CC cells. miRNAs serve important roles in cell growth, cell differentiation, cell apoptosis, cell cycle and other physiological and pathological processes by binding to the 3'-UTR of target genes, thus, regulating its expression of mRNA and protein level. According to TargetScan 7.1, miRDB and microRNA.org, ST7L is a predicted target gene of miR-378 (Fig. 3A). To verify that miR-378 can directly target ST7L mRNA, EGFP reporter 
A

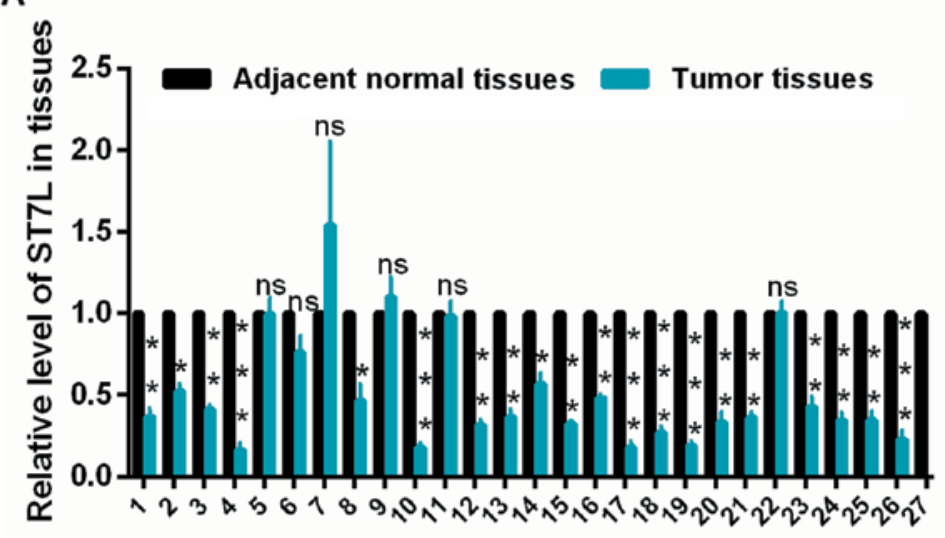

C
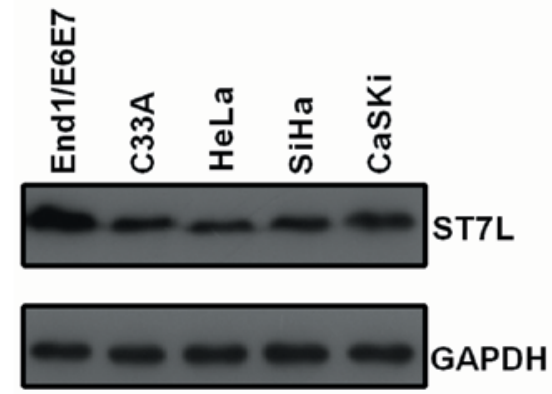

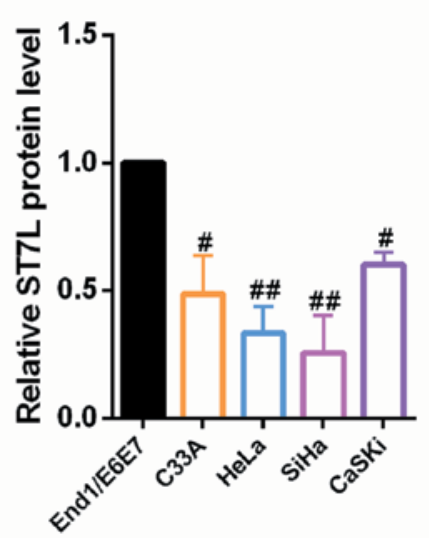

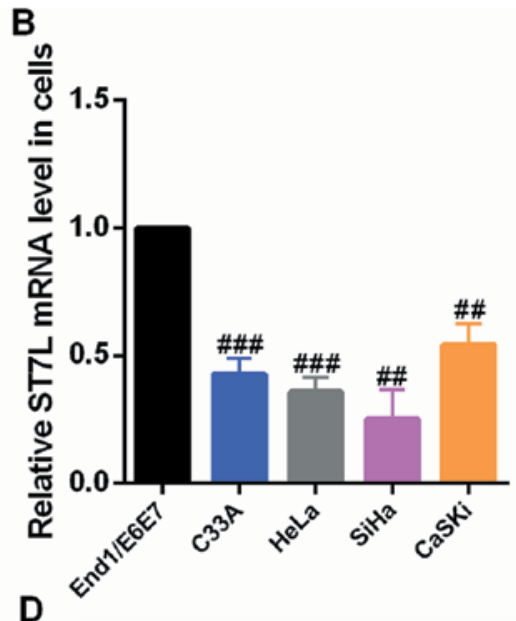

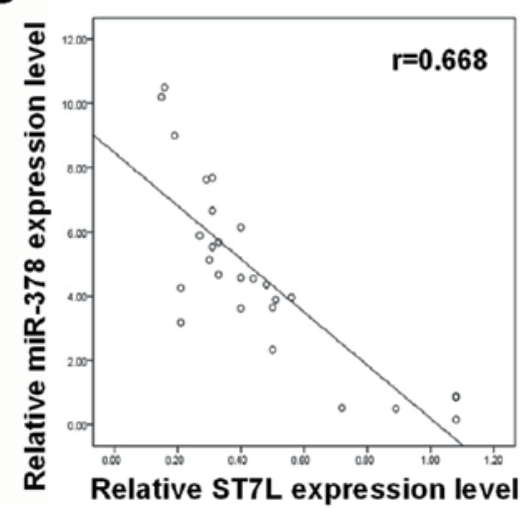

Figure 4. The mRNA level of ST7L was downregulated in CC tissues and CC cells. (A) The mRNA levels of ST7L in CC and normal tissues were examined by RT-qPCR assay. (B) The mRNA levels of ST7L in C33A, HeLa, SiHa, CaSKi cells and End1/E6E7 cells were examined by RT-qPCR assay. (C) The protein levels of ST7L in C33A, HeLa, SiHa and CaSKi cells and End1/E6E7 cells were examined by western blot assay. (D) The correlation of the expression of miR-378 and ST7L in tumor tissues was presented using SPSS 19.0. Data are presented as mean $\pm \mathrm{SD}(\mathrm{n}=3)$. ${ }^{*} \mathrm{P}<0.05,{ }^{* *} \mathrm{P}<0.01,{ }^{* * * *} \mathrm{P}<0.001$ vs. adjacent normal tissues; ${ }^{\#} \mathrm{P}<0.05,{ }^{\# \#} \mathrm{P}<0.01,{ }^{\# \# \#} \mathrm{P}<0.001$ vs. the End1/E6E7 cells. CC, cervical cancer; RT-qPCR, reverse transcription-quantitative polymerase chain reaction; miR, microRNA.

plasmids containing ST7L wild-type or mutant 3'-UTR were constructed. In HeLa and SiHa cells, the relative EGFP activity was significantly reduced in both the pri-miR-378 and wild-type 3'-UTR of ST7L co-transfected group and co-transfection with ASO-miR-378 and wild-type 3'-UTR of ST7L increased the relative EGFP activity (Fig. 3B). However, miR-378 overexpression or knockdown did not play a role in EGFP activity regulated by the 3'-UTR of ST7L mutation (Fig. 3C). In addition, significant downregulation of ST7L was observed at both mRNA and protein levels in HeLa and SiHa cells expressing pri-miR-378 and the upregulation of ST7L at both mRNA and protein levels in the cells expressing ASO-miR-378 (Fig. 3D and E).

miR-378 promotes the malignancy of CC by inhibiting ST7L expression. First, the authors detected the mRNA level of ST7L by RT-qPCR in 27 pairs of cervical tumors and matched normal cervical tissues and in cervical cancer cells and human normal cervical epithelium cell. The mRNA level of ST7L was significantly decreased in human cervical cancer tissues (Fig. 4A) and cervical cancer cells (Fig. 4B) compared with the control groups. As presented in Fig. 4C, the protein level of ST7L was lower in CC cell lines than in End1/E6E7 cells by western blot assay. Moreover, the expression level of ST7L was negatively correlated with the expression level of miR-378 in tumor tissues (Fig. 4D). The overexpression or knockdown plasmid of ST7L was effective in HeLa and SiHa cell lines (Fig. 5A). Then, authors performed an MTT assay and colony formation assay using cells transfected with pST7L or shR-ST7L and the control plasmids. At 24, 48 and $72 \mathrm{~h}$ posttransfection, HeLa and SiHa cell viability were decreased by overexpression of ST7L and increased by ST7L knockdown (Fig. 5B). The relative colony formation rate was decreased by pST7L and increased by shR-ST7L compared to the control groups (Fig. 5C). The cell cycle assay demonstrated that ST7L overexpression increased the number of HeLa and SiHa cells in the G1 phase, decreased the number in the S phase (Fig. 5D), and ST7L overexpression was observed to significantly promote cell apoptosis in HeLa and SiHa cells (Fig. 5E). The noted effect of ST7L on apoptosis was confirmed, because increased activating cleavage of PARP and caspase- 3 was induced in the cervical cancer cells that overexpressed ST7L (Fig. 5F).

Upregulated miR-378 activates the Wnt/ $\beta$-catenin pathway in cervical cancer cells. Inactivation of $\mathrm{Wnt} / \beta$-catenin signaling caused by ST7L by repressing $\beta$-catenin expression could explain this phenomenon to a certain extent in epithelial ovarian cancer (21). To address these issues, the expression of Wnt genes and the activity of $\beta$-catenin were analyzed in the 

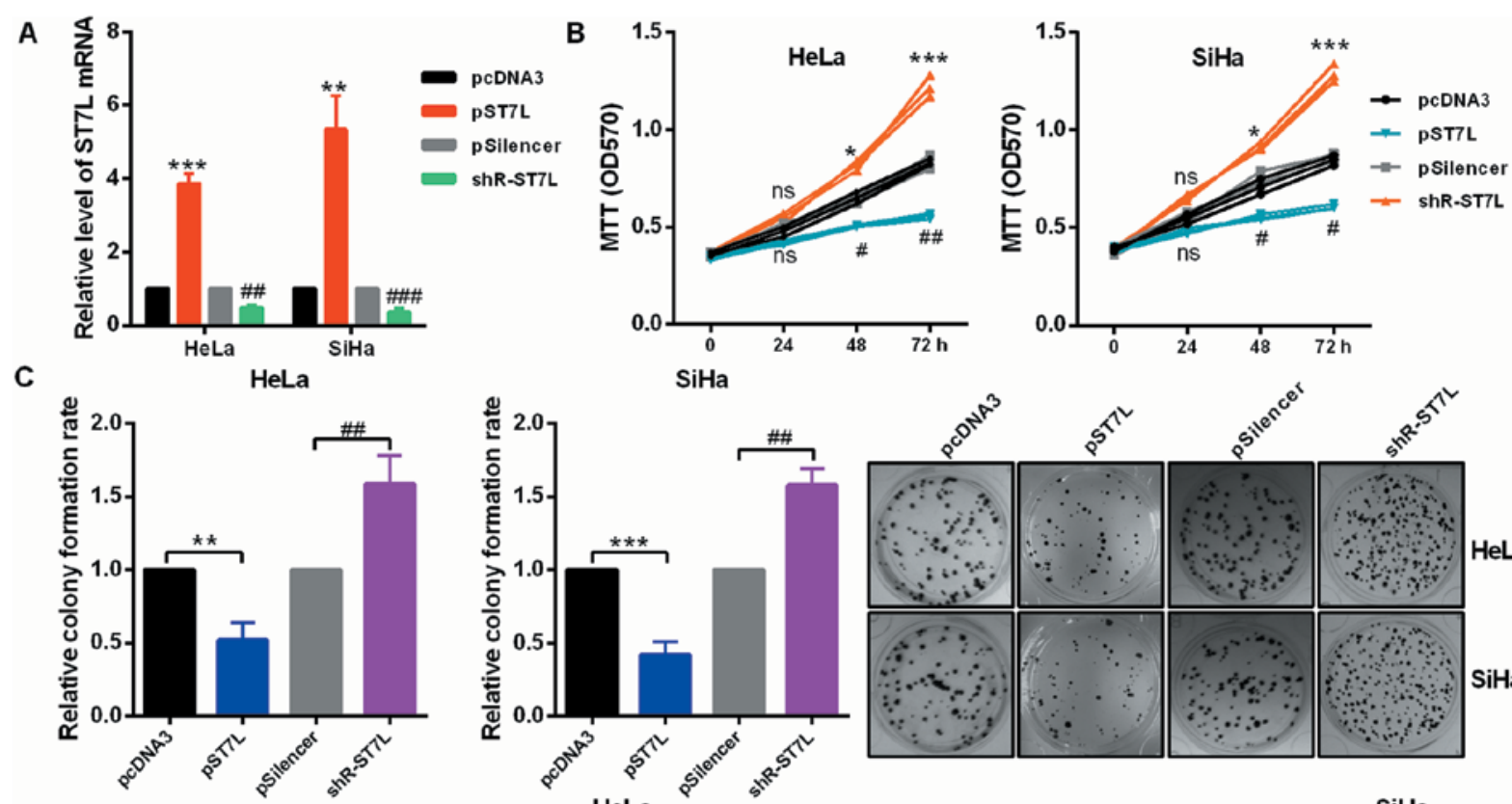

D
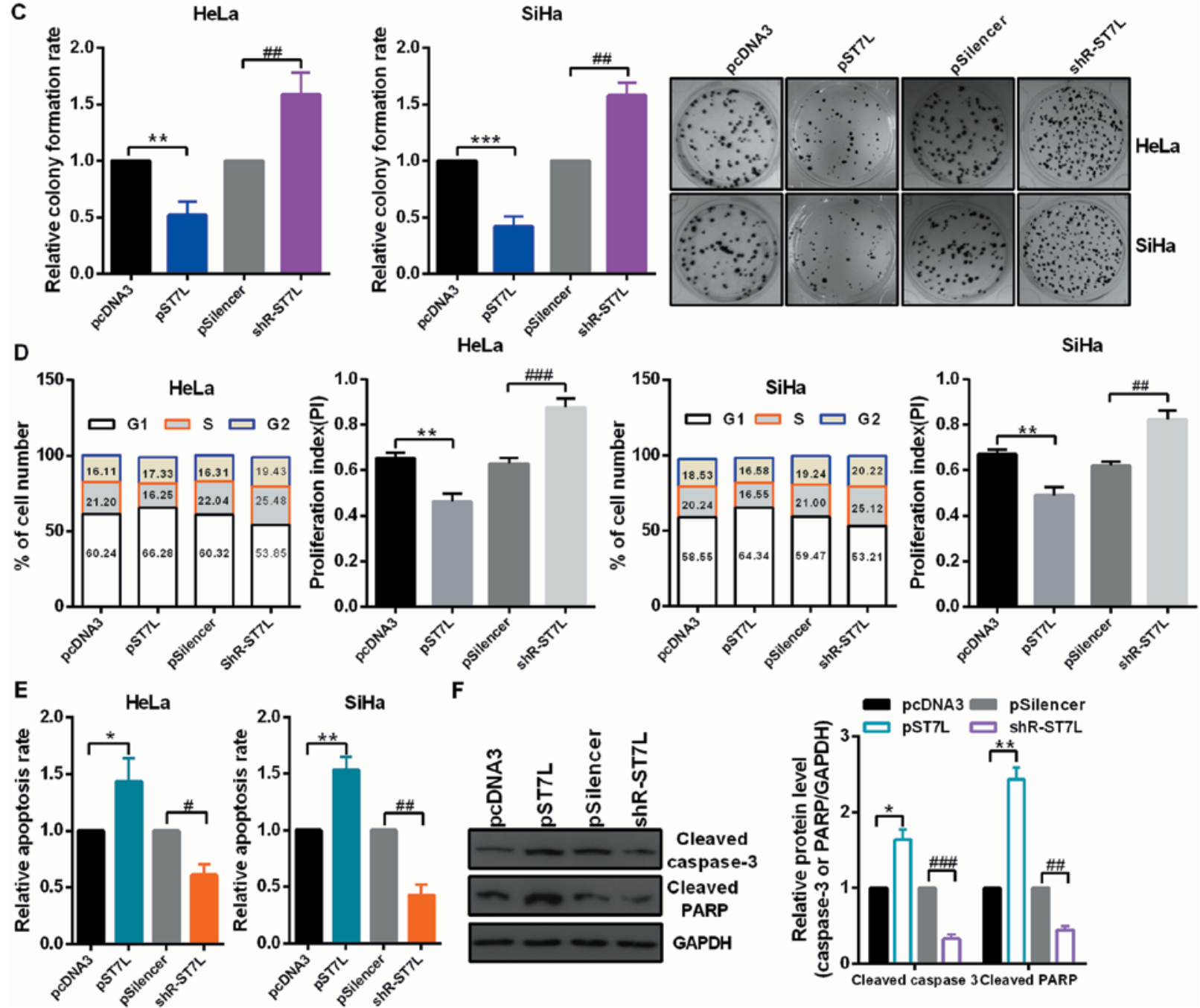

Figure 5. ST7L functions as a tumor suppressor gene in cervical cancer cells. (A) Reverse transcription-quantitative polymerase chain reaction assay indicated that overexpression and knockdown of ST7L in HeLa and SiHa were efficient. (B) MTT assay showed that overexpression of ST7L inhibited cell viability and knockdown of ST7L increased cell viability in HeLa and SiHa cells. (C) Colony formation rates were lower following transfection with pST7L and higher following transfection with shR-ST7L compared with the control groups. Original magnification was x1. (D) The cell cycle profiles after treatment with pST7L or shR-ST7L were detected by flow cytometry assay. (E) Cell apoptosis assay showed that overexpression of ST7L promoted the apoptosis and that knockdown of ST7L inhibited the apoptosis of HeLa and SiHa cells. (F) Western blot analysis showed that overexpression of ST7L increased the levels of cleaved caspase-3 and PARP and the silencing of ST7L had the opposite effect. Data are presented as mean $\pm \mathrm{SD}(\mathrm{n}=3) .{ }^{*} \mathrm{P}<0.05,{ }^{* *} \mathrm{P}<0.01,{ }^{* * * *} \mathrm{P}<0.001 \mathrm{vs}$. the pcDNA3 group; ${ }^{"} \mathrm{P}<0.05$, ${ }^{\# \#} \mathrm{P}<0.01,{ }^{\# \# \#} \mathrm{P}<0.001$ vs. the pSilencer group.

context of miR-378 overexpression or miR-378 overexpression in conjunction with pST7L in cervical cancer cells. Western blot assays showed that the expression of activated $\beta$-catenin, $\mathrm{C}$-myc and cyclin $\mathrm{D}$, which are the downstream effectors of the Wnt/ $\beta$-catenin pathway, were reduced by ST7L depletion and significantly increased by miR-378 restoration (Fig. 6A). The localization of $\beta$-catenin in HeLa cells was examined by immunofluorescence after transfection with pri-miR-378 or pST7L or co-transfection with pri-miR-378 and pST7L. The nuclear distribution of $\beta$-catenin was increased in primiR-378-transfected cells and decreased in pST7L-transfected cells (Fig. 6B). In addition, TOP/FOP luciferase reporter assays were also performed, which is a common Wnt pathway activation reporter assay, and the results demonstrated that miR-378 enhanced the TOP/FOP flash ratio but ST7L weakened it (Fig. 6C). 
A
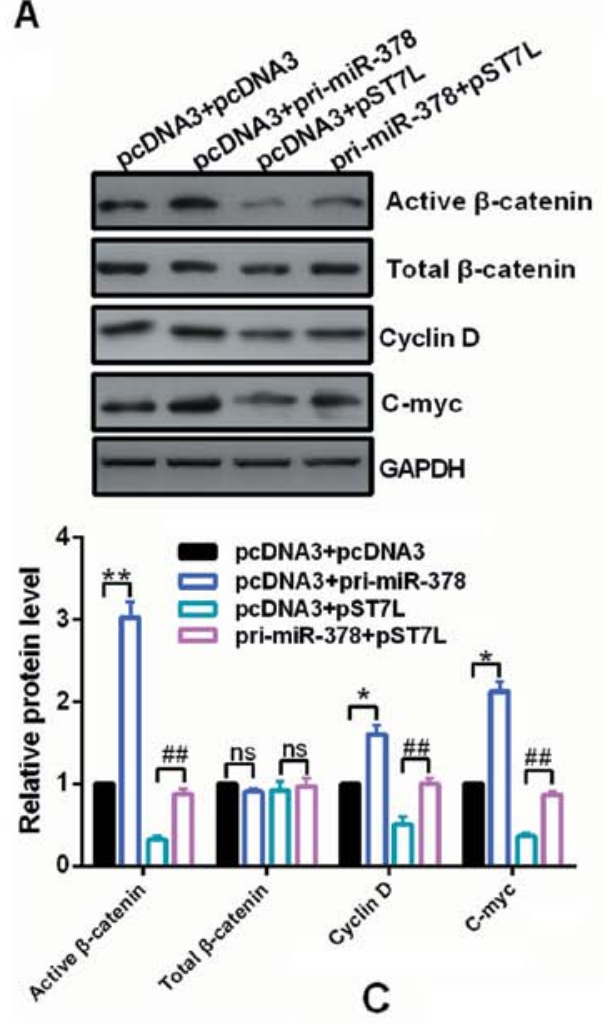

B
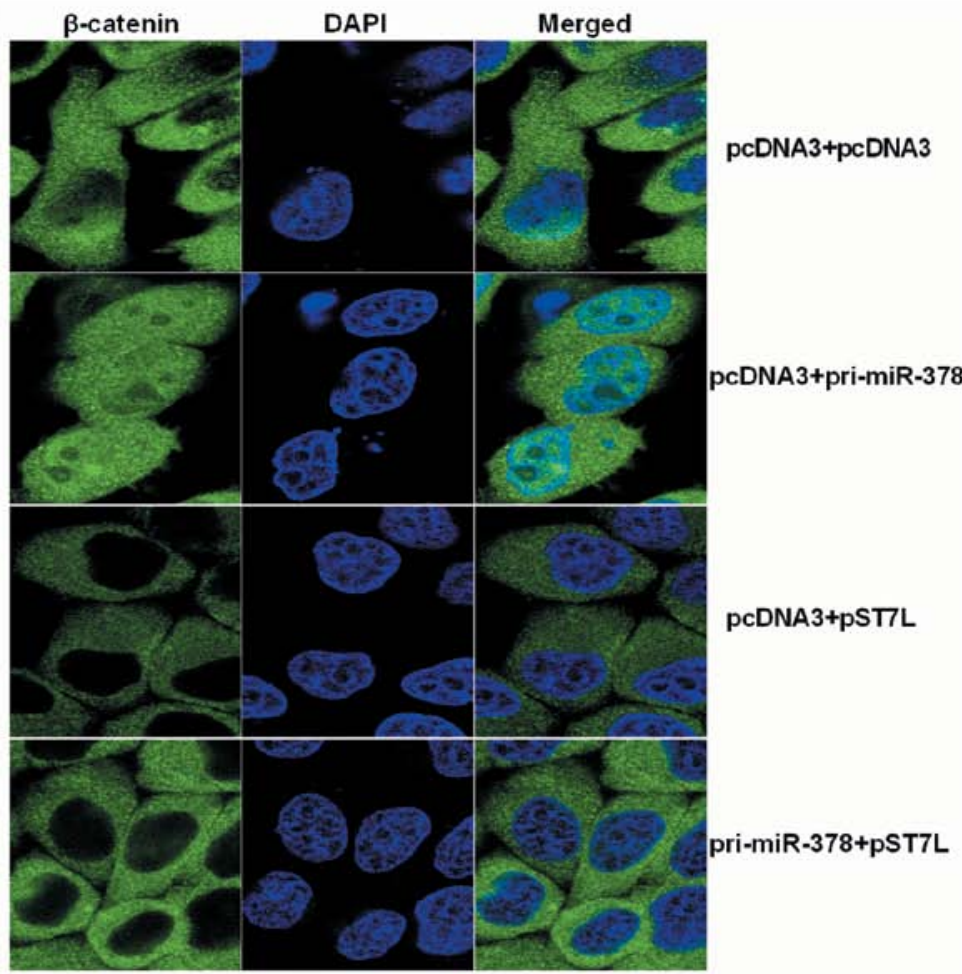

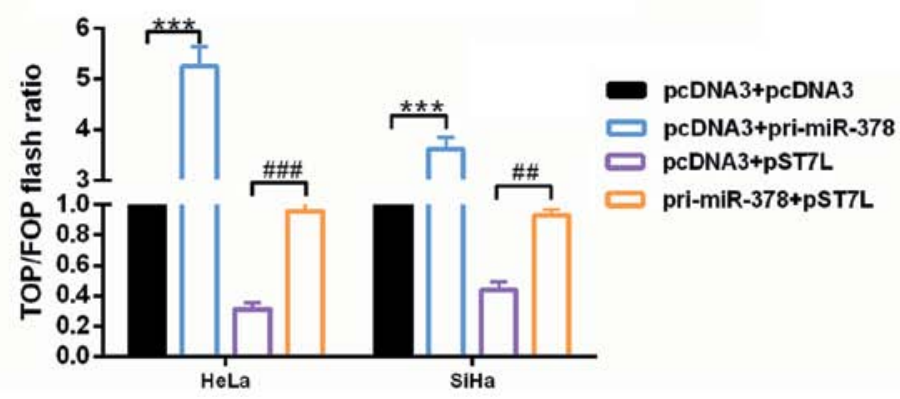

Figure 6. miR-378 positively regulates the Wnt/ $\beta$-catenin pathway. (A-C) HeLa cells were transfected with the indicated combinations of pri-miR-378, pST7L, pri-miR-378 and pST7L or the control groups. (A) Western blot assay was used to detect the expression level of $\beta$-catenin, C-myc and cyclin D. (B) Immunofluorescence assay was used to examine the nuclear distribution of $\beta$-catenin. Original magnification was x1000. (C) Top/Fop luciferase reporter assays were performed to detect the $\beta$-catenin activity. Data are presented as mean $\pm \mathrm{SD}(\mathrm{n}=3) .{ }^{*} \mathrm{P}<0.05,{ }^{* *} \mathrm{P}<0.01,{ }^{* * *} \mathrm{P}<0.001$ vs. the pcDNA3+pcDNA3 group; ${ }^{\# \#} \mathrm{P}<0.01,{ }^{\# \#} \mathrm{P}<0.001$ vs. the pcDNA3+pST7L group. miR, microRNA; pri, primary.

\section{Discussion}

Previously, miRNAs have appeared as a highlighted class of gene regulators and dysregulation of miRNAs plays important roles in the genesis and development of various cancers including cervical cancer (23-25). Reports have indicated that miR-378 is overexpressed in many cancers (26). For example, upregulated miR-378 enhanced cell proliferation, cell migration and cell invasion in HepG2 cells, and promoted the metastasis of the tumor cells to the liver (10). In addition, overexpression of miR-378 increased neural stem cell (NSC) differentiation and reduced NSC proliferation, whereas suppression of miR-378 led to decreased NSC differentiation and increased NSC proliferation and miR-378 negatively regulated Tailless mRNA and protein expression (14). Furthermore, miR-378 levels significantly decreased in N2A cells following oxygen-glucose deprivation treatment and overexpression of miR-378 significantly enhanced cell viability, decreased cell apoptosis and the immunoreactivity of cleaved-caspase- 3 by targeting the predicted 3 '-UTR of caspase- 3 gene in the ischemic injury (27). However, Chen et al (28) reported that miR-378 suppresses cell growth by downregulating MAPK1 expression in prostate cancer; and $\mathrm{Li}$ et al (29) demonstrated that the expression level of miR-378 is obviously lower in glioma tissues compared with normal brain tissues. In addition, Li et al (30) also concluded that miR-378 may synergistically act with curcumin in inhibiting cell growth by binding to the 3'-UTR of p38 in glioblastoma cells. These studies suggested that miR-378 serves important roles in multiple types of human cancer and may therefore be a therapeutic target in their treatment. Thus, to investigate the role of miR-378 in various cancers is a matter of great account. In the present study, miR-378 was significantly upregulated in both CC tissues and cell lines. Functional assays demonstrated that miR-378 overexpression promoted cell proliferation, accelerated the cell cycle process and reduced apoptosis in cervical 
cancer cells. More importantly, miR-378 overexpression could activate the $\mathrm{Wnt} / \beta$-catenin pathway in $\mathrm{CC}$ cells. However, the mechanism that regulates the expression of miR-378 in $\mathrm{CC}$ tissues and cells is not well understood; therefore, the detail mechanism needs further investigation.

It is well known that miRNAs can play roles by relying on the level of complementarities with the 3'-UTR of their target mRNAs (31), the authors next attempted to identify the potential targets of miR-378. Bioinformatics software was used to predict the target genes of miR-378, in the present study, ST7L was demonstrated to be a novel candidate target of miR-378. Notably, the mRNA expression level of ST7L was significantly decreased in HeLa, SiHa, C33A and Caski cell lines, and CC tissues, which was inversely correlated with miR-378 level in CC tissues and cells. Furthermore, the EGFP reporter assay presented that miR-378 binds directly to the 3'-UTR of the ST7L mRNA in HeLa and SiHa cells. To examine the regulatory roles of miR-378 on endogenous ST7L expression, RT-qPCR and western blot analyses were utilized to measure ST7L expression at the mRNA and protein levels in CC cells following transfection with pri-miR-378 or ASO-miR-miR-378. Results revealed that miR-378 overexpression markedly reduced the expression of ST7L at mRNA and protein level and miR-378 knockdown increased the expression of ST7L at mRNA and protein level in HeLa and SiHa cells.

ST7L, also as known as ST7R, STLR and FAM4B, was confirmed with its similarity to the ST7 tumor suppressor gene found in the chromosome 7q31 region and clustered with the WNT2B gene in a tail-to-tail manner in a chromosomal region known to be deleted and rearranged in many cancers, including germ cell tumors, breast cancer and non-small cell lung cancer, amongst others $(18,19)$. Furthermore, ST7L was reported to inhibit the Wnt/ $\beta$-catenin signaling pathway and act as a tumor-suppressor gene in many cancers (20-22). However, the function and role of ST7L in CC cells was unclear. The current results demonstrated that ST7L was downregulated in $\mathrm{CC}$ tissues and cells. Functional assays revealed that overexpression of ST7L suppressed cell proliferation by arresting the cell cycle process and promoted cell apoptosis in HeLa and SiHa cells. In addition, ST7L inhibited the Wnt/ $\beta$-catenin pathway in CC cells.

In conclusion, these reports confirmed that upregulation of miR-378 is a common phenomenonin CC tissues and CC cells, and the authors have identified that miR-378 takes effect in regulating cell proliferation, cell cycle, cell apoptosis and the Wnt/ $\beta$-catenin pathway of CC cells. miR-378 inhibits ST7L expression at both the mRNA and protein level by directly targeting the 3'-UTR of the ST7L transcript. With its regulation of cervical cancer cell malignant phenotype, knockdown of miR-378 functions as a novel therapeutic strategy in CC.

\section{References}

1. Jemal A, Bray F, Center MM, Ferlay J, Ward E and Forman D Global cancer statistics. CA Cancer J Clin 61: 69-90, 2011.

2. Bansal N, Herzog TJ, Shaw RE, Burke WM, Deutsch I and Wright JD: Primary therapy for early-stage cervical cancer: Radical hysterectomy vs radiation. Am J Obstet Gynecol 201: 485.e1-485.e9, 2009.
3. Kosmas C, Mylonakis N, Tsakonas G, Vorgias G, Karvounis N, Tsavaris N, Daladimos T, Kalinoglou N, Malamos N, Akrivos T, et al: Evaluation of the paclitaxel-ifosfamide-cisplatin (TIP) combination in relapsed and/or metastatic cervical cancer. Br J Cancer 101: 1059-1065, 2009.

4. Colombo N, Carinelli S, Colombo A, Marini C, Rollo D and Sessa C; ESMO Guidelines Working Group: Cervical cancer: ESMO Clinical Practice Guidelines for diagnosis, treatment and follow-up. Ann Oncol 23 (Suppl 7): vii27-vii32, 2012.

5. Castellsagué X, Díaz M, de Sanjosé S, Muñoz N, Herrero R, Franceschi S, Peeling RW, Ashley R, Smith JS, Snijders PJ, et al; International Agency for Research on Cancer Multicenter Cervical Cancer Study Group: Worldwide human papillomavirus etiology of cervical adenocarcinoma and its cofactors: Implications for screening and prevention. J Natl Cancer Inst 98: 303-315, 2006.

6. Wang Z, Cai H, Lin L, Tang M and Cai H: Upregulated expression of microRNA-214 is linked to tumor progression and adverse prognosis in pediatric osteosarcoma. Pediatr Blood Cancer 61: 206-210, 2014.

7. Tamura M, Uyama M, Sugiyama Y and Sato M: Canonical Wnt signaling activates miR-34 expression during osteoblastic differentiation. Mol Med Rep 8: 1807-1811, 2013.

8. Davis-Dusenbery BN and Hata A: Mechanisms of control of microRNA biogenesis. J Biochem 148: 381-392, 2010.

9. Chan JK, Kiet TK, Blansit K, Ramasubbaiah R, Hilton JF, Kapp DS and Matei D: MiR-378 as a biomarker for response to anti-angiogenic treatment in ovarian cancer. Gynecol Oncol 133: $568-574,2014$

10. Ma J, Lin J, Qian J, Qian W, Yin J, Yang B, Tang Q, Chen X, Wen X, Guo H, et al: MiR-378 promotes the migration of liver cancer cells by down-regulating Fus expression. Cell Physiol Biochem 34: 2266-2274, 2014.

11. Browne G, Dragon JA, Hong D, Messier TL, Gordon JA, Farina NH, Boyd JR, VanOudenhove JJ, Perez AW, Zaidi SK, et al: MicroRNA-378-mediated suppression of Runx1 alleviates the aggressive phenotype of triple-negative MDA-MB-231 human breast cancer cells. Tumour Biol 37: 8825-8839, 2016.

12. Yu BL, Peng XH, Zhao FP, Liu X, Lu J, Wang L, Li G, Chen HH and Li XP: MicroRNA-378 functions as an onco-miR in nasopharyngeal carcinoma by repressing TOB2 expression. Int $\mathrm{J}$ Oncol 44: 1215-1222, 2014.

13. Qian J, Lin J, Qian W, Ma JC, Qian SX, Li Y, Yang J, Li JY, Wang CZ, Chai HY, et al: Overexpression of miR-378 is frequent and may affect treatment outcomes in patients with acute myeloid leukemia. Leuk Res 37: 765-768, 2013.

14. Huang Y, Liu X and Wang Y: MicroRNA-378 regulates neural stem cell proliferation and differentiation in vitro by modulating Tailless expression. Biochem Biophys Res Commun 466: 214-220, 2015.

15. Peng J, Xie Z, Cheng L, Zhang Y, Chen J, Yu H, Li Z and Kang H: Paired design study by real-time PCR: miR-378* and miR-145 are potent early diagnostic biomarkers of human colorectal cancer. BMC Cancer 15: 158, 2015.

16. Liu H, Zhu L, Liu B, Yang L, Meng X, Zhang W, Ma Y and Xiao H: Genome-wide microRNA profiles identify miR-378 as a serum biomarker for early detection of gastric cancer. Cancer Lett 316: 196-203, 2012

17. Fedorko M, Stanik M, Iliev R, Redova-Lojova M, Machackova T, Svoboda M, Pacik D, Dolezel J and Slaby O: Combination of MiR-378 and MiR-210 serum levels enables sensitive detection of renal cell carcinoma. Int J Mol Sci 16: 23382-23389, 2015.

18. Katoh M: Molecular cloning and characterization of ST7R (ST7-like, ST7L) on human chromosome 1p13, a novel gene homologous to tumor suppressor gene ST7 on human chromosome 7q31. Int J Oncol 20: 1247-1253, 2002.

19. Kirikoshi H and Katoh M: Expression of ST7R (ST7-like, ST7L) in normal tissues and cancer. Int J Oncol 21: 193-196, 2002.

20. Chen L, Zhang A, Li Y, Zhang K, Han L, Du W, Yan W, Li R, Wang Y, Wang K, et al: MiR-24 regulates the proliferation and invasion of glioma by ST7L via $\beta$-catenin/Tcf- 4 signaling. Cancer Lett 329: 174-180, 2013.

21. Yang Z, Wang XL, Bai R, Liu WY, Li X, Liu M and Tang H: miR-23a promotes IKK $\alpha$ expression but suppresses ST7L expression to contribute to the malignancy of epithelial ovarian cancer cells. Br J Cancer 115: 731-740, 2016.

22. Zhuang L, Wang X, Wang Z, Ma X, Han B, Zou H, Wu Z, Dong S, Qu Z, Zang Y, et al: MicroRNA-23b functions as an oncogene and activates AKT/GSK $3 \beta / \beta$-catenin signaling by targeting ST7L in hepatocellular carcinoma. Cell Death Dis 8: e2804, 2017. 
23. Bartel DP: MicroRNAs: Genomics, biogenesis, mechanism, and function. Cell 116: 281-297, 2004.

24. Park H, Lee MJ, Jeong JY, Choi MC, Jung SG, Joo WD, Lee C and An HJ: Dysregulated microRNA expression in adenocarcinoma of the uterine cervix: Clinical impact of miR-363-3p. Gynecol Oncol 135: 565-572, 2014.

25. Schickel R, Boyerinas B, Park SM and Peter ME: MicroRNAs: Key players in the immune system, differentiation, tumorigenesis and cell death. Oncogene 27: 5959-5974, 2008.

26. Li ZZ, Shen LF, Li YY, Chen P and Chen LZ: Clinical utility of microRNA-378 as early diagnostic biomarker of human cancers: A meta-analysis of diagnostic test. Oncotarget 7: 58569-58578, 2016.

27. Zhang N, Zhong J, Han S, Li Y, Yin Y and Li J: MicroRNA378 alleviates cerebral ischemic injury by negatively regulating apoptosis executioner caspase-3. Int J Mol Sci 17: E1427, 2016.
28. Chen QG, Zhou W, Han T, Du SQ, Li ZH, Zhang Z, Shan GY and Kong CZ: MiR-378 suppresses prostate cancer cell growth through downregulation of MAPK1 in vitro and in vivo. Tumour Biol 37: 2095-2103, 2016.

29. Li B, Wang Y, Li S, He H, Sun F, Wang C, Lu Y, Wang X and Tao B: Decreased expression of miR-378 correlates with tumor invasiveness and poor prognosis of patients with glioma. Int $\mathbf{J}$ Clin Exp Pathol 8: 7016-7021, 2015.

30. Li W, Yang W, Liu Y, Chen S, Chin S, Qi X, Zhao Y, Liu H, Wang J, Mei X, et al: MicroRNA-378 enhances inhibitory effect of curcumin on glioblastoma. Oncotarget: May 16, 2017 (Epub ahead of prin). dooi: 10.18632/oncotarget.17881.

31. Farazi TA, Juranek SA and Tuschl T: The growing catalog of small RNAs and their association with distinct Argonaute/Piwi family members. Development 135: 1201-1214, 2008. 